

\title{
Mars as a comet: Solar wind interaction on a large scale
}

\author{
Mats Holmstrom, Xiao-Dong Wang \\ Swedish Institute of Space Physics, PO Box 812, SE-98128 Kiruna, Sweden
}

\begin{abstract}
Looking at the Mars-solar wind interaction on a larger spatial scale than the near Mars region, the planet can be seen as an ion source interacting with the solar wind, in many ways like a comet, but with a smaller ion source region. Here we study the interaction between Mars and the solar wind using a hybrid model (particle ions and fluid electrons). We find that the solar wind is disturbed by Mars out to 100 Mars radii downstream of the planet, and beyond. On this large scale it is clear that the escaping ions can be classified into two different populations. A polar plume of ions picked-up by the solar wind, and a more fluid outflow of ions behind the planet. The outflow increases linear with the production up to levels of observed outflow rates, then the escape levels off for higher production rates.
\end{abstract}

Keywords: Mars, Solar wind interaction

\section{Introduction}

The interaction between the solar wind and Mars provides one way that the atmosphere of Mars can be lost. As soon as neutrals in Mars atmosphere are ionized, e.g., by photoionization, they are subject to electro-magnetic forces and 5 can be accelerated and subsequently escape the planet. To quantify the present loss of atmosphere at Mars, it is therefore of interest to understand how ions can escape the planet under different conditions. In the past, ion outflow has been pbserved by the Phobos-2 (Lundin et al. 1989) and the Mars Express (Lundin

Email address: matsh@irf.se (Mats Holmstrom)

Preprint submitted to Planetary and Space Science

September 3, 2015

(C) 2015. This manuscript version is made available under the Elsevier user license http://www.elsevier.com/open-access/userlicense/1.0/ 
et al. 2004) missions. In the most general sense, the loss of ions from Mars can be seen as a mass loading (Szegö et al., 2000) of the solar wind, similar in some ways to the mass loading at comets, but with a smaller ion source region. If we go to even larger spatial scales, the fluxes of ions produced near Mars will be too tenuous to affect the solar wind, and behave like an expanding cloud of ions. Here we focus on the intermediate region, far away from the planet, but not so far away that the solar wind is unperturbed. What is the morphology of the ion outflow, and the global interaction of the planet with the solar wind? What is the morphology of the bow shock in the far wake region? The tool we use is a hybrid model (particle ions and fluid electrons) of the interaction between Mars and the solar wind, that we describe in the next section.

The solar wind interaction with Mars has been modeled by many groups using different kinds of models, e.g., empirical (Kallio and Luhmann, 1997) test particles (Fang et al., 2008), magnetohydrodynamic (MHD) (Ma et al., 2004), multi-fluid (Harnett and Winglee, 2006) and hybrid models (Brecht, 1990, Kallio and Janhunen, 2001; Modolo et al., 2005, Bößwetter et al., 2007).

The advantage of fluid models is that they are computationally less expensive than hybrid models, allowing finer grid resolution. On the other hand, hybrid models contains a more accurate description of the ion physics, something important at Mars where the planet is of similar size as the ion length scales, such as the ion gyro radius and the ion inertial length. We can note that so a comparison of many different models of the interaction of Mars with the solar wind found significant differences in the general interaction for the different models (Brain et al. 2010).

One thing in common to past model investigations is that they have consider the near Mars region, up to a few planet radii away from the planet. This is 35 natural since the aim of the studies has been focused on topics such as determining the loss of heavy ions to space. This requires that the model resolves the ionospheric region well with high spatial resolution. This implies a high computational cost for a large simulation domain, so the domain is chosen just large enough for the process under study. For ion escape studies the simulation 
40 ion flux.

Previous studies has found that the escaping ions can be classified into two different populations. A polar plume of ions picked-up by the solar wind, and a more fluid outflow of ions behind the planet. In hybrid simulations Brecht

45 and Ledvina (2012) found tailward electric fields in the hemisphere opposite to the solar wind convective electric field the accelerate heavy ions such that they escape downstream behind the planet. The ion plume has been described based on Mars Express observations (Liemohn et al. 2014), and has also been studied using test particle simulations (Curry et al., 2013).

\section{Model}

In what follows we describe the details of the computer model we use to study the large scale plasma interaction between Mars and the solar wind. The model has previous been applied to the solar wind interactions of the Moon (Holmström et al. 2012) and the plasma interactions of Callisto (Lindkvist et al. 2015). First

55 we describe the plasma model, then the heavy ion production, and finally the boundary conditions.

\subsection{Hybrid model}

In the hybrid approximation, ions are treated as particles, and electrons as a massless fluid. In what follows we use SI units. The trajectory of an ion, $\mathbf{r}(t)$ 60 and $\mathbf{v}(t)$, with charge $q$ and mass $m$, is computed from the Lorentz force,

$$
\frac{d \mathbf{r}}{d t}=\mathbf{v}, \quad \frac{d \mathbf{v}}{d t}=\frac{q}{m}(\mathbf{E}+\mathbf{v} \times \mathbf{B})
$$

where $\mathbf{E}=\mathbf{E}(\mathbf{r}, t)$ is the electric field, and $\mathbf{B}=\mathbf{B}(\mathbf{r}, t)$ is the magnetic field. From now on we do not write out the dependence on $\mathbf{r}$ and $t$. The electric field is given by

$$
\mathbf{E}=\frac{1}{\rho_{I}}\left(-\mathbf{J}_{I} \times \mathbf{B}+\mu_{0}^{-1}(\nabla \times \mathbf{B}) \times \mathbf{B}-\nabla p_{e}\right)+\frac{\eta}{\mu_{0}} \nabla \times \mathbf{B},
$$


where $\rho_{I}$ is the ion charge density, $\mathbf{J}_{I}$ is the ion current density, $p_{e}$ is the electron

pressure, $\eta$ is the resistivity, and $\mu_{0}=4 \pi \cdot 10^{-7}$ is the magnetic constant. Then Faraday's law is used to advance the magnetic field in time,

$$
\frac{\partial \mathbf{B}}{\partial t}=-\nabla \times \mathbf{E} .
$$

Note that the unknowns are the position and velocity of the ions, and the magnetic field on a grid, not the electric field, since it can always be computed from (1). Further details on the hybrid model used here, and the discretization, 70 can be found in (Holmström, 2011, Holmstrom, 2011, Holmström et al., 2012).

In the wake of obstacles to the solar wind flow, e.g., behind Mars, the plasma densities can be low, or even zero. In such regions of low ion charge density, $\rho_{I}$, the hybrid method can have numerical problems. We see from (1) that the electric field computation involves a division by $\rho_{I}$, so regions of low density

75 will have large electric fields. This can lead to numerical instabilities, due to large gradients in the electric field, and due to large accelerations of ions. The numerical solution quickly becomes unstable. Here we handle regions of low ion charge density by solving a magnetic diffusion equation in such regions. A magnetic diffusion equation is also solved inside the obstacle to the solar wind flow, in this case inside the spherical inner boundary. See Holmstrom (2013) for details of this approach.

\subsection{Ion production}

Instead of building a complete ionospheric model with many species, sources, and loss terms, we only specify a source of photoions given by a standard Chap85 man production function (Kivelson and Russell, 1995).

$$
p(h, \chi)=p_{0} e^{1-y-\sec \chi e^{-y}}, \quad y=\left(h-h_{0}\right) / H, \quad h \geq 0, \quad 0 \leq \chi<\pi / 2,
$$

where $h[\mathrm{~m}]$ is the height above the planet surface, $\chi[\mathrm{rad}]$ is the solar zenith angle (SZA), $p_{0}\left[\mathrm{~m}^{-3} \mathrm{~s}^{-1}\right]$ is the maximum production along the sub-solar line, at height $h_{0}[\mathrm{~m}]$, and $H[\mathrm{~m}]$ is the scale height. In this study we only consider atomic oxygen, $\mathrm{O}^{+}$, ions, and we call them heavy ions (heavies) as opposed to 
the other species in the simulation, solar wind protons, $\mathrm{H}^{+}$, and solar wind alpha particles, $\mathrm{He}^{++}$. This is a very simplified ionospheric model. It only contains one heavy ion specie, has an unrealistic ion production profile, and does not contain any ion chemical reactions.

The justification for such a simplified ionospheric model is that we are interested in the effects on large spatial scales, where we can view Mars as a source of ions in the solar wind and the exact details of the distribution of heavy ions close to the planet should not be very important.

After selecting $h_{0}$ and $H$ we can then choose different $p_{0}$ that will result in different outflow rates of heavy ions. Since the outflow rate of heavy ions has been observed to be on the order of $10^{25} \mathrm{~s}^{-1}($ Ramstad et al. 2013) we choose a production $p_{0}$ that gives an outflow on that order of magnitude.

\subsection{Boundary conditions}

Heavy ions will be lost from the simulation in two ways. Either through the inner spherical boundary, or through the outer boundary of the simulation box. Such ions are removed from the simulation.

One interesting feature of the simulations is that the whole magnetosphere becomes unstable after some time if the simulation cells are larger than about $1000 \mathrm{~km}$. The bow shock then flares out and can hit the upstream boundary of the simulation domain.

\subsection{Parameter values}

Unless otherwise noted, the parameter values used in the simulation runs are as follows.

The coordinate system is centered at Mars, with the $x$-axis directed toward the Sun, so the solar wind flows opposite to the $x$-axis. The radius of Mars, $R_{M}=3380 \mathrm{~km}$.

We have used typical solar wind conditions at Mars from Brain et al. (2010). In the upstream solar wind the interplanetary magnetic field (IMF) is $(-1.634,2.516,0.0) \mathrm{nT}$ with a magnitude of $3 \mathrm{nT}$. This direction of the IMF is along the nominal Parker 
spiral at Mars. The solar wind speed is $400 \mathrm{~km} / \mathrm{s}$, with a proton number den-

120 The solar wind proton temperature is $5 \cdot 10^{4} \mathrm{~K}$, and the electron temperature is $1.2 \cdot 10^{5} \mathrm{~K}$. For these plasma parameters in the solar wind, the ion inertial length is $131 \mathrm{~km}$, the Alfvén velocity is $38 \mathrm{~km} / \mathrm{s}$, the thermal proton gyro radius is $100 \mathrm{~km}$ (the gyro time is $22 \mathrm{~s}$ ), and the ion plasma beta is 0.6 . height of $h_{0}=500 \mathrm{~km}$. The scale height, $H=250 \mathrm{~km}$. Note that the scale and peak heights of the ionosphere are much larger than in reality. However, this does not affect the results much since the cell size of the simulations are larger than these heights. Thus, the ionosphere is not resolved at all in the simulations. not change the results much. The weight of the heavy ion macroparticles are $4 \cdot 10^{20}$ (this many $\mathrm{O}^{+}$ions per macroparticle. We keep the produced number of heavy ion macroparticles constant. So if $p_{0}$ is increased by a factor, then the weights of the heavy ion macroparticles are reduced by the same factor. The boundary conditions on the other faces are periodic. The simulation domain is divided into a Cartesian grid with cubic cells of size $700 \mathrm{~km}$. The extent of the simulation box is $[-186.6,15] \times[-100.8,100.8] \times[-100.8,100.8] \cdot 10^{6} \mathrm{~km}$ $\approx[-55.2,4.44] \times[-29.8,29.8]^{2} R_{M}$. At the inflow boundary we have three $\mathrm{H}^{+}$ simulation macroparticles is about 100 million. Five subcycles of the cyclic leapfrog (CL) algorithm (Holmström, 2011) are used here when updating the magnetic field, and the execution time is about 24 hours on $432 \mathrm{CPU}$ cores.

\section{Results}

We now investigate the results for four different relative heavy ion productions; $0.01,0.1,1$, and 10 . The morphology of the heavy ion outflow and the magnetic field magnitude is shown in Figure1. It is seen that the heavy ion out- 


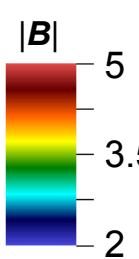

$[\mathrm{nT}]$
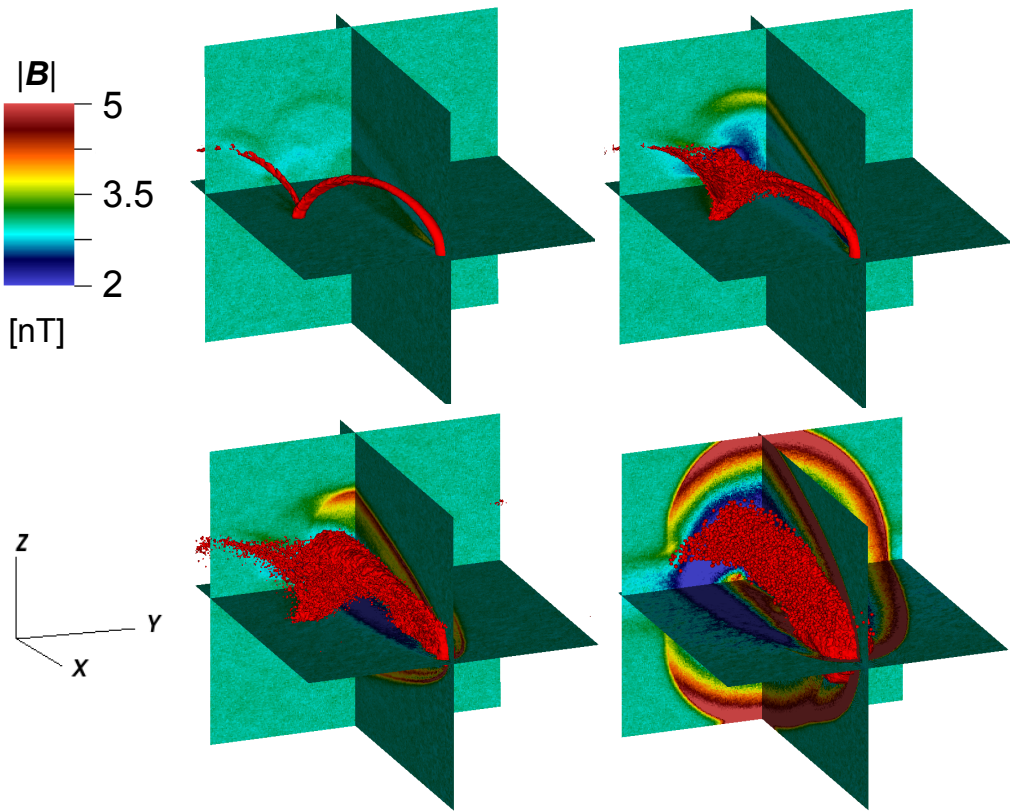

Figure 1: Results for relative heavy ion production of 0.01 (top left), 0.1 (top right), 1 (bottom left), and 10 (bottom right). Iso surface of heavy ion number density of $0.01 \mathrm{~cm}^{-3}$ is shown in red. The colors of the cuts show magnetic field strength according to the color bar. The simulation domain extends to $50 R_{M}$ down stream of Mars, and the solution is at $t=1200 \mathrm{~s}$. The cut perpendicular to the $x$-axis is at $x=-1.6 \cdot 10^{8} \mathrm{~m}$.

flow follows two different channels. A pick-up like escape where the ions gyrate in the interplanetary magnetic field (IMF) and a more fluid escape downstream of the planet. At the lowest production of 0.01 all the heavy ions are picked-up. They are still present in the higher production cases (0.1 and 1) but then there is also escape behind Mars. For the highest production of 10, all ions escape fluid like in the tail behind Mars. However, the simulation had not reached a steady state yet for the highest production. For the magnetic field, we can see in Figure 1 that for the lowest production of 0.01 no significant bow shock is formed, there are however field disturbances associated with the picked-up ions. 


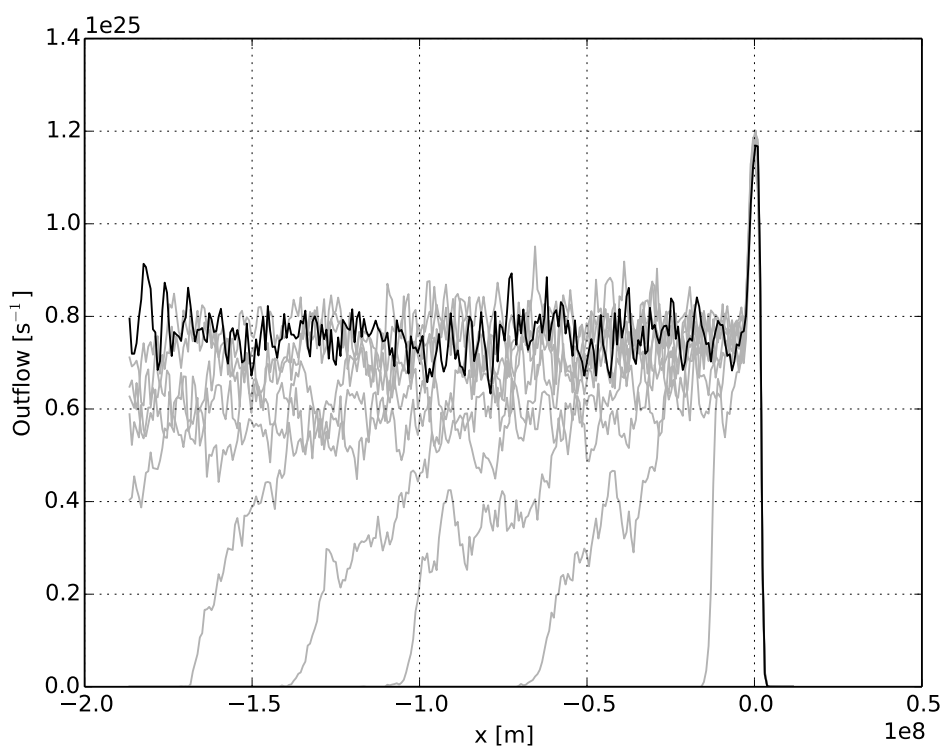

Figure 2: Total $\mathrm{O}^{+}$number flux through planes perpendicular to the $x$-axis for a relative heavy ion production of 1 , at different times. The black line is at the final time of $1100 \mathrm{~s}$, and the grey lines are at earlier times in intervals of $100 \mathrm{~s}$.

For larger production a bow shock is formed that strengthen with increased production. We also see that the outflowing ions generate field disturbances downstream that resembles a break in the bow shock,

For the production 1 case we show in Figure 2 the total $\mathrm{O}^{+}$number flux as a function of distance downstream of Mars, for different simulation times. We see that the simulation reaches a steady state situation after 900 seconds.

In Figure 3 we show the average velocity of $\mathrm{O}^{+}$ions as a function of distance downstream of Mars. It takes 45 Mars radii before the heavy ions reach the solar wind velocity of $400 \mathrm{~km} / \mathrm{s}$. Visible is also the gyration of some of the ions, as a drop in velocity at about $-30 R_{M}$. This is due to the cycloid motion of the picked-up heavy ions.

We now examine how the outflow changes with production. In Table 1 we show the total outflow of $\mathrm{O}^{+}$ions for different normalized production values. 
Figure 3: The average velocity of $\mathrm{O}^{+}$ions in planes perpendicular to the $x$-axis for a relative heavy ion production of 1 , after 1100 seconds. The velocity is computed as the total ion current divided by total charge in each plane.

Table 1: Total outflow of $\mathrm{O}^{+}$ions as a function of normalized production.

\begin{tabular}{l|l|l|l} 
Production & 0.01 & 0.1 & 1 \\
\hline Outflow $\left[\mathrm{s}^{-1}\right]$ & $2.8 \cdot 10^{23}$ & $2.6 \cdot 10^{24}$ & $0.7 \cdot 10^{25}$
\end{tabular}

not reach a steady state after $1200 \mathrm{~s}$. The heavy ions accumulated over time close to the planet and were not transported away to escape. The outflow is determined as an average behind Mars at a time of steady state, as shown in Figure 2 for the case when the production is 1 . We see in Table 1 that the outflow increases linear with the production for low productions (from 0.01 to 0.1 ). For higher productions (from 0.1 to 1 ) the outflow levels off.

In Fig. 4 we show the magnetic field strength and the heavy ions for a run out to $100 R_{M}$ downstream of Mars. On this large scale, we clearly see the mix of two different populations of escaping $\mathrm{O}^{+}$. One population is the picked-up ${ }_{180} \mathrm{O}^{+}$that is visible to the left, moving in cycloid motion perpendicular to the 


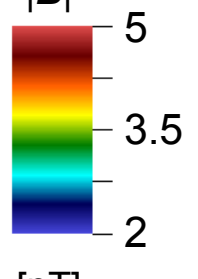

[nT]

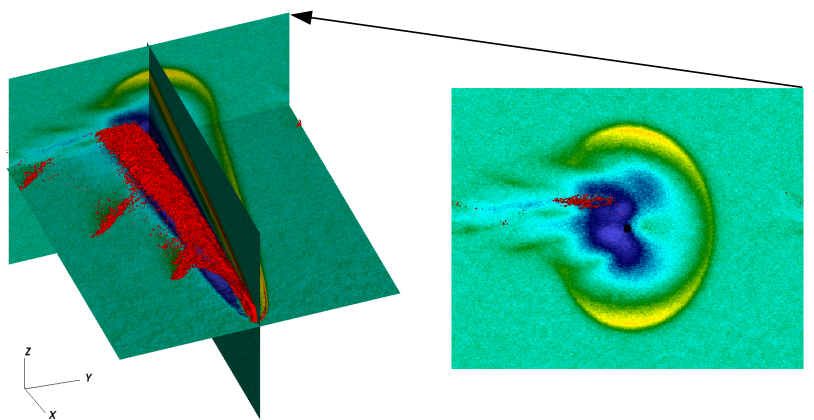

Figure 4: Results for a relative heavy ion production of 1 . Iso surface of heavy ion number density of $0.02 \mathrm{~cm}^{-3}$ is shown in red. The colors of the cuts show magnetic field strength according to the color bar. The simulation domain extends to $100 R_{M}$ down stream of Mars, and the solution is at $t=1200 \mathrm{~s}$. The cut perpendicular to the $x$-axis is at $x=-3.4 \cdot 10^{8} \mathrm{~m}$, and the black disk at the center is the projection of Mars onto this plane.

IMF. The other population is a more fluid-like bulk flow of heavy ions going down stream in the $-x$-direction. We also see how the Mach cone is disturbed and penetrated by the escaping heavy ions. The bulk flow is several $R_{M}$ above the plane of the IMF, and is not extended much even at this far distance from the planet. There is some spreading in the plane of the IMF (the $x y$-plane), but not much in the direction perpendicular to the IMF plane.

For the simulation out to $100 R_{M}$ downstream of Mars, we also show the magnetic field magnitude and the proton number density in Fig. 5 On this scale the bow shock is non-existent in the plane of the IMF on the side where the bow shock normal would be quasi parallel to the IMF. There are however disturbances associated with the gyrating heavy ions. We can also see that the bow shock is split on the quasi perpendicular side, as has been observed closer to Mars in earlier simulations (Modolo et al., 2005). This is seen also in the proton number density, along with also disturbances associated with the gyrating heavy ions. The protons are slowed down close to the planet and in 


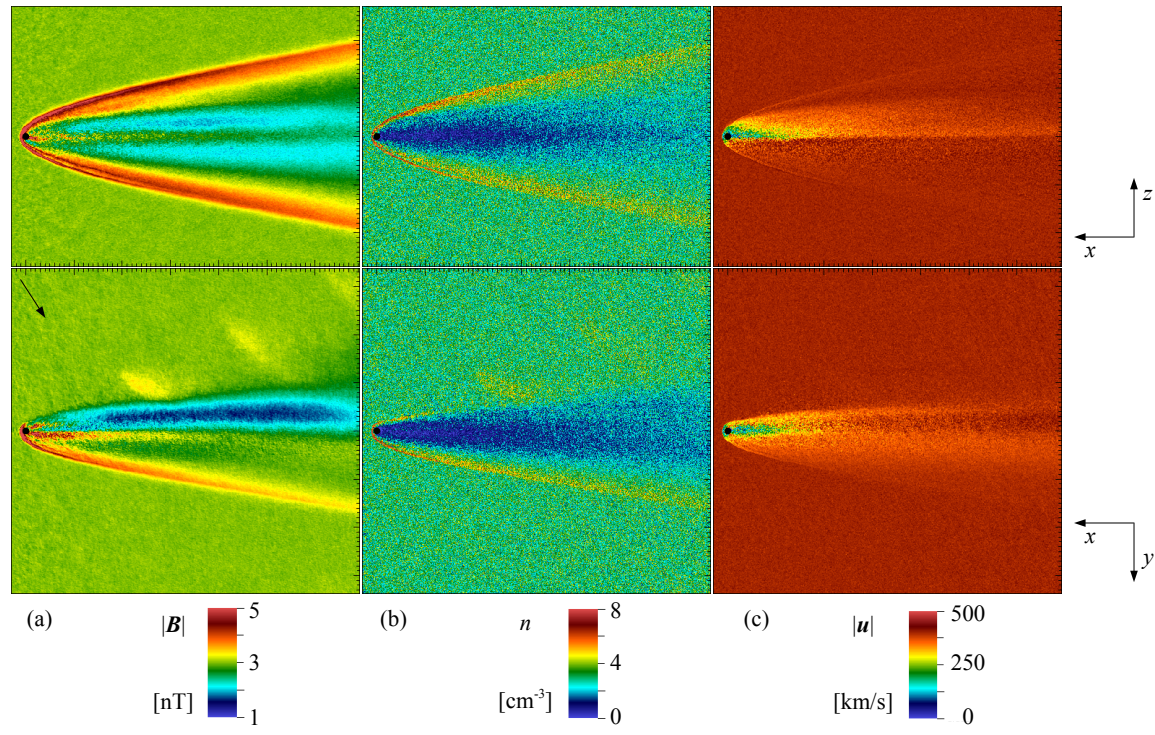

Figure 5: Magnetic field magnitude (a), $\mathrm{H}^{+}$number density (b), and $\mathrm{H}^{+}$velocity magnitude (c), for a relative heavy ion production of 1 . Cuts in the $x z$-plane (top) and in the $x y$-plane (bottom). The direction of the interplanetary magnetic field (IMF) in the $x y$-plane is indicated by a black arrow. The simulation domain extends to $100 R_{M}$ down stream of Mars, and the solution is at $t=1200 \mathrm{~s}$. The black disk is the projection of Mars. The maximum magnetic field magnitude is $9.3 \mathrm{nT}$ and the maximum number density is $15 \mathrm{~cm}^{-3}$. 
the wake, but are accelerated to solar wind speeds further down stream.

\section{Conclusions}

We have studied the interaction of Mars with the solar wind on a large scale, out to 100 Mars radii downstream of the planet. A simplified ionosphere 5256 , and was conducted using resources provided by the Swedish National Infrastructure for Computing (SNIC) at the High Performance Computing Center North (HPC2N), Umeå University, Sweden. The software used in this work was 
in part developed by the DOE NNSA-ASC OASCR Flash Center at the University of Chicago.

\section{References}

Bößwetter, A., et al., 2007. Comparison of plasma data from ASPERA-3/MarsExpress with a 3-D hybrid simulation. Annales Geophysicae 25, 1851-1864.

Brain, D., et al., 2010. A comparison of global models for the solar wind interaction with Mars. Icarus 206, 139-151.

Brecht, S.H., 1990. Magnetic asymmetries of unmagnetized planets. Geophysical Research Letters 17, 1243-1246.

Brecht, S.H., Ledvina, S.A., 2012. Control of ion loss from mars during solar minimum. Earth Planets Space, 64, 165178, 2012 , 165-178.

235 Curry, S.M., et al., 2013. The influence of production mechanisms on pick-up ion loss at Mars. Journal of Geophysical Research 118, 554-569.

Fang, X., et al., 2008. Pickup oxygen ion velocity space and spatial distribution around Mrs. Journal of Geophysical Research 113, A02210.

Harnett, E.M., Winglee, R.M., 2006. Three-dimensional multi-fluid simulations of ionospheric loss at Mars from nominal solar wind conditions to magnetic cloud events. Journal of Geophysical Research 111, A09213.

Holmstrom, M., 2011. An energy conserving parallel hybrid plasma solver, in: Proceedings of ASTRONUM-2010, pp. 211-216. ArXiv:1010.3291.

Holmström, M., 2011. Hybrid modeling of plasmas, in: Proceedings of ENUMATH 2009, the 8th European Conference on Numerical Mathematics and Advanced Applications, Springer. pp. 451-458. ArXiv:0911.4435.

Holmstrom, M., 2013. Handling vacuum regions in a hybrid plasma solver, in: Proceedings of ASTRONUM-2012, pp. 202-207. ArXiv:1301.0272. 
Holmström, M., Fatemi, S., Futaana, Y., Nilsson, H., 2012. The interaction between the moon and the solar wind. Earth, Planets and Space 64, 237-245. doi:10.5047/eps.2011.06.040

Kallio, E., Janhunen, P., 2001. Atmospheric effects of proton precipitation in the Martian atmosphere and its connection to the Mars-solar wind interaction. Journal of Geophysical Research 106, 5617-5634.

Kallio, E., Luhmann, J., 1997. Charge exchange near Mars: The solar wind absorption and energetic neutral atom production. Journal of Geophysical Research 102, 22183-22197.

Kivelson, M.G., Russell, C.T. (Eds.), 1995. Introduction to Space Physics. Cambridge. Page 187.

Liemohn, M.W., Johnson, B.C., Fränz, M., Barabash, S., 2014. Mars Express observations of high altitude planetary ion beams and their relation to the energetic plume loss channel. Journal of Geophysical Research 119, 97029713.

Lindkvist, J., Holmström, M., Khurana, K.K., Fatemi, S., Barabash, S., 2015. Callisto plasma interactions: Hybrid modeling including induction by a subsurface ocean. Journal of Geophysical Research: Space Physics 120, 48774889.

Lundin, R., et al., 1989. First results of the ionospheric plasma escape from Mars. Nature 341, 609-612.

Lundin, R., et al., 2004. Solar wind-induced atmospheric erosion at Mars: First results from ASPERA-3 on Mars Express. Science 305, 1993-1936.

Ma, Y., et al., 2004. Three-dimensional, multispecies, high spatial resolution MHD studies of the solar wind interaction with Mars. Journal of Geophysical Research 109, A07211. 
275 Modolo, R., et al., 2005. Influence of the solar EUV flux on the Martian plasma environment. Annales Geophysicae 23, 433-444.

Ramstad, R., et al., 2013. Phobos 2/ASPERA data revisited: Planetary ion escape rate from Mars near the 1989 solar maximum. Geophysical Research Letters 40, 477-481.

280 Szegö, K., et al., 2000. Physics of mass loaded plasmas. Space Science Reviews 94, 429-671. doi 10.1023/A:1026568530975. 